\title{
Pathologic response after modern radiotherapy for non-small cell lung cancer
}

\author{
Simon F. Roy ${ }^{1}$, Alexander V. Louie ${ }^{2}$, Moishe Liberman ${ }^{3}$, Philip Wong ${ }^{4}$, Houda Bahig ${ }^{4}$ \\ ${ }^{1}$ Department of Pathology, University of Montreal, Montreal, QC, Canada; ${ }^{2}$ Department of Radiation Oncology, Sunnybrook Health Sciences \\ Centre, Toronto, ON, Canada; ${ }^{3}$ Division of Thoracic Surgery, Department of Surgery, ${ }^{4}$ Department of Radiation Oncology, Centre Hospitalier de \\ l'Université de Montréal, Montreal, QC, Canada \\ Contributions: (I) Conception and design: SF Roy, H Bahig; (II) Administrative support: None; (III) Provision of study materials or patients: None; (IV) \\ Collection and assembly of data: SF Roy, AV Louie, H Bahig; (V) Data analysis and interpretation: All authors; (VI) Manuscript writing: All authors; \\ (VII) Final approval of manuscript: All authors. \\ Correspondence to: Dr. Houda Bahig, MD, PhD. Department of Radiation Oncology, Centre Hospitalier de l'Université de Montréal, 1051 Sanguinet \\ Street, Montreal, QC, Canada. Email: houdabahig@gmail.com.
}

\begin{abstract}
In non-small cell lung cancer (NSCLC), pathologic complete response (pCR) following radiotherapy treatment has been shown to be an independent prognostic factor for long-term survival, progression-free survival and locoregional control. PCR is considered a surrogate to therapeutic efficacy, years before survival data are available, and therefore can be used to guide treatment plans and additional therapeutic interventions post-surgical resection. Given the extensive fibrotic changes induced by radiotherapy in the lung, radiological assessment of response can potentially misrepresent pathologic response. The optimal timing for assessment of pathologic response after conventionally fractionated radiotherapy and stereotactic ablative radiotherapy (SABR) remains poorly understood. In this review, we summarize recent literature on pathologic response after radiotherapy for early stage and locally advanced NSCLC, we discuss current controversies around radiobiological considerations, and we present upcoming trials that will provide insight into current knowledge gaps.
\end{abstract}

Keywords: Stereotactic ablative radiotherapy (SABR); non-small cell lung cancer (NSCLC); pathologic complete response (pCR)

Submitted Aug 28, 2019. Accepted for publication Sep 02, 2019.

doi: 10.21037/tlcr.2019.09.05

View this article at: http://dx.doi.org/10.21037/tlcr.2019.09.05

\section{Introduction}

In non-small cell lung cancer (NSCLC), pathologic complete response (pCR) following neoadjuvant chemoradiotherapy (CRT) has been shown to be an independent prognostic factor for progression-free survival, overall survival (OS) and locoregional control (1). In addition to providing insight as to treatment efficacy, accurately recognizing complete response in both early stage and locally advanced NSCLC (LA-NSCLC) is important for defining patient prognosis, determining the need for further adjuvant therapy, and guiding optimal follow-up evaluation (2). However, pathologic response after lung radiotherapy has been reported in only a small number of trials, most frequently in the setting of a historical interest for neoadjuvant irradiation in locally advanced lung cancer. In the context of stereotactic ablative radiotherapy (SABR) in early stage NSCLC, given that the majority of patients offered this treatment are not surgical candidates, the literature on pathologic response has, until recently, been inexistent. In addition, the disparity in pCR definitions across trials further complicates the interpretation of outcomes. In fact, there is a range of definitions of pCR, varying between the absence of viable tumor cells within a surgically-resected specimen to the presence of complete fibrosis or necrosis, as assessed by conventional histology 
upon light microscopy (3). Hellmann et al. proposed the use of the term "major pathologic response", defined as $10 \%$ or less residual viable tumour, as another surrogate endpoint given its association with OS (3). Pataer and colleagues observed that among patients with NSCLC treated with neoadjuvant chemotherapy, each additional percentage of viable tumor post treatment was associated with a $1 \%$ increase in the risk of death (4). The Swiss Group for Clinical Cancer Research had defined pCR as "necrosis more than or equal to $95 \%$ and fibrosis on pathologic examination" (5).

In the current review, we will discuss the results from the recent MISSILE-NSCLC ("Measuring the Integration of Stereotactic Radiotherapy Plus Surgery in Early NonSmall Cell Lung Cancer") phase II trial on pathologic response after neoadjuvant SABR in early-stage NSCLC (6) as well as the major studies reporting pathologic response after conventionally fractionated radiotherapy +/- systemic therapy in LA-NSCLC. Current controversies on the optimal timing and methods for assessment of pathologic response, considering radiobiological mechanisms, will be discussed. Finally, on-going trials of combined radiotherapy and immunotherapy in the neoadjuvant setting will be reviewed.

\section{The case of early stage NSCLC}

\section{Challenges of radiological assessment of response after $S A B R$}

SABR is effectively the gold standard treatment approach in patients with inoperable stage I NSCLC, with local control rates as high as $90 \%$ at 5 years (7-14). The role of SABR in operable or marginally operable patients is currently being assessed in the context of several on-going randomized controlled trials (NCT02984761, NCT02629458, NCT01753414, NCT02468024).

One of the main challenges in monitoring outcomes after SABR in inoperable patients is distinguishing radiological peri-tumoral fibrotic changes from tumor recurrence $(6,15,16)$. As such, $50 \%$ of patients treated with SABR will show signs of radiological progression on computed tomography (CT) imaging following SABR (17), while 90\% will develop late fibrotic changes (18-20) at a median time of 4 months and frequently after 1 year (18-20), making radiological evaluation of response unreliable. Therefore, the Response Evaluation Criteria in Solid Tumors (RECIST) version 1.1 criteria, which rely strictly on tumor dimension changes, are not optimal for response assessment post-SABR (21). To address this gap, retrospective studies and systemic reviews have identified radiologic predictors of disease persistence and/or progression, which include a combination of morphological findings on serial CT along with post-SABR maximum standardized uptake value $\left(\mathrm{SUV}_{\max }\right) \geq 5.0$ on 18 -fluorodeoxyglucose positron emission tomography (18-FDG-PET) (16,22-24). While no definitive guidelines exist, the use of these predictors has been widely adopted in the clinical setting as it is considered the best available strategy to distinguish post-SABR parenchymal changes from disease persistence and/or progression (25). In this radiological approach, persistent enlargements on CT may not count as local failure, which may lead to overestimation of local control rates. In addition, concurrent benign inflammatory glucose uptake that can occur up to 12 months post-SABR complicates 18-FDG-PET interpretation (15). In view of these limitations, pathologic assessment of response after SABR remains the gold standard to evaluate the absence of viable tumor cells. In the context of the growing role of SABR, better understanding of the histological changes underlying the radiological observations, for clearer insight into treatment efficacy, is essential for optimization of subsequent therapies and follow-up.

\section{Evidence for pathologic response post SABR}

In the hopes of increasing treatment potency, decreasing positive margin rates, and gaining understanding of the expected pathologic responses, the group from London Health Sciences Centre undertook a phase 2 clinical trial aiming at examining pCR rate after neoadjuvant SABR in operable early stage NSCLC patients (Table 1) (6). The MISSILE-NSCLC trial enrolled 40 operable patients with histologically confirmed clinical T1-T2aN0 NSCLC treated with standard dose lung SABR (54-60 Gy in 3-8 fractions, corresponding to biologically effective dose $>100 \mathrm{~Gy}_{10}$ ), followed by surgery (lobectomy or sublobar resection) at a median time of 10 weeks (range, 9-16 weeks) post-SABR completion (6). A lobectomy or sublobar resection was performed in $90 \%$ of patients, along with sampling of hilar and mediastinal lymph nodes; $10 \%$ of patients did not undergo surgery due to radiation-induced pneumonitis, poor performance status, pulmonary function precluding operability, or regional disease progression (6).

Tumor cell viability was assessed by standard 
Table 1 Pathologic complete response rates after neoadjuvant SABR for resectable early-stage NSCLC

\begin{tabular}{|c|c|c|c|c|c|c|c|}
\hline Author & Trial name & Year & $\begin{array}{l}\text { Treatment } \\
\text { regimen }\end{array}$ & $\mathrm{N}$ surgically-resected ${ }^{\star}$ & pCR rate & $\begin{array}{l}\text { Time between } \\
\text { neoadjuvant therapy } \\
\text { and resection }\end{array}$ & Comment \\
\hline \multirow{2}{*}{$\begin{array}{l}\text { Palma } \\
\text { et al. }\end{array}$} & \multirow{2}{*}{$\begin{array}{c}\text { MISSILE-NSCLC } \\
\text { (NCT02136355) }\end{array}$} & \multirow{2}{*}{2019} & \multirow{2}{*}{$\begin{array}{l}\text { Neoadjuvant } \\
\text { SABR plus } \\
\text { surgery }\end{array}$} & \multirow{2}{*}{35} & \multirow{2}{*}{$60 \%$} & \multirow{2}{*}{10 weeks } & 2-year local control: $100 \%$ \\
\hline & & & & & & & 2-year regional control: $53 \%$ \\
\hline
\end{tabular}

*, one pathology specimen was improperly fixated in formalin and thus could not be analyzed. SABR, stereotactic ablative radiotherapy; $\mathrm{N}$, number of patients; NSCLC, non-small cell lung cancer; pCR, pathologic complete response.

hematoxylin-eosin staining and morphologic appearance of tumor cells on microscopy. While a pCR rate of $90 \%$ was hypothesized based on historical local control outcomes of lung SABR, the investigators reported a pCR rate of only $60 \%$ at 10 weeks post-SABR. At 8 weeks post-SABR, he corresponding complete and partial radiological responses were $2 \%$ (1 patient) and $43 \%$ (17 patients), respectively. In surgically operated patients, 2-year OS, local control, regional control and distant control was $77 \%, 100 \%$, $53 \%$ and $76 \%$, respectively. While the reported pCR may seem strikingly inconsistent with the high clinical local control observed in previous studies, there are multiple possible underlying explanations. The kinetics of postSABR pathologic response are largely unknown, and many have hypothesized that the ultimate pCR rate could be underestimated at 10 weeks (26). This is consistent with current radiobiological models supporting that in addition to apoptosis, post-mitotic death is a major mechanism of cellular death after radiotherapy. In this model, unrepaired DNA damage leads to cell inactivation during mitosis, with a critical level of genomic instability reached sometimes only after several cycles of cell division (27-30). In this context, tumor cells can appear viable on histopathology but in fact are dead, dying, or senescent from lethal chromosomal damage. With the caveat of different treatment techniques and modality combination, this is analogous to previous evidence in anal canal cancer treated with CRT, where the optimal time for assessment of response was found to be at 26 weeks post-treatment, as many patients with partial response at 11 weeks finally developed complete response by 26 weeks (31). Increased pCR rates with increasing time from radiotherapy completion have also been reported in rectal and esophageal cancers (32-34). However, it is unclear if these findings can be directly translated to SABR, where the radiobiological effect is thought to also involve vascular damage and deterioration of the intratumor environment leading to tumor cell death (35). It is however possible that early assessment of pathologic response at 10 weeks postSABR is poorly representative of the actual radiotherapy damage and clonogenic survival.

On the other hand, an alternative explanation is that pCR is indeed lower than expected after lung SABR. In fact, in a subgroup analysis by median time from the end of SABR to surgery ( $<74 v s . \geq 74$ days), pCR remained $60 \%$ in both groups. The high local control rates of SABR reported in the literature are derived from largely inoperable patients population with multiple comorbidities, and one may argue that these rates are subject to competing risk of death from other causes. In addition, as previously stated, radiological assessments of local control with current methods may underestimate disease persistence due to the confounding fibrotic changes. In a recent orthotopic model of NSCLC in rats, Oweida et al. induced NSCLC in 11 rats, of which 5 were assigned to observation and 6 received a SABR dose of 34 Gy in one fraction (36). Animals were sacrificed at different time points $(10,30$ and 60 days postSABR) to evaluate radiologic and histologic responses to SABR longitudinally. Radiologically, 4/6 animals had CR with disappearance of tumor on imaging within 30 days of therapy, 1 had partial response and 1 had radiologic progression. Interestingly, radiologic responses were found to match the observed pathologic responses: the 4 animals with CR had radiation-induced pneumonitis upon histology, and moderately differentiated mucinous adenocarcinoma was present in the two tumors that showed either partial response or progression (36).

Current SABR literature as well as our current knowledge of radiobiological effects of radiotherapy suggest 
that the outcomes from MISSILE likely underestimate the actual pCR after SABR and should be considered with caution. However, MISSILE certainly highlights the critical need for better understanding of the pathological efficacy of SABR and reiterates that SABR alone should be used with vigilance in operable patients outside of the on-going clinical trials.

\section{The case of locally advanced NSCLC}

The current standard treatment in patients with LANSCLC (stage IIIA/IIIB) is concurrent CRT followed by adjuvant durvalumab based on the recently published PACIFIC trial (37). In comparison to SABR, pathologic response is more extensively reported in LA-NSCLC given the multiple previous trials that studied neoadjuvant approaches in this patient's population with the aim of improving the historically dismal outcomes. These past studies typically included highly heterogenous groups of patients and generally showed no survival advantage to the addition of surgery to CRT. However, selected patients may have improved outcomes from neoadjuvant treatments and, in the era of immunotherapy, this approach is now being revisited in on-going clinical trials.

\section{Pathologic response after neoadjuvant radiotherapy in locally advanced NSCLC}

Data on pathologic response after conventional radiotherapy alone in LA-NSCLC is provided by the historical use of neoadjuvant radiotherapy followed by surgical resection (38-43) (Table 2). The Lung Cancer Study Group (LCSG 881) compared preoperative radiotherapy (44 Gy in 22 fractions) and preoperative chemotherapy (mitomycin, vinblastine and cisplatin) in a total of 67 stage III patients. The median survival was 12 months in both arms, and only 1 patient developed pCR in each arm (41). The randomized control trial from the Cancer and Leukemia Group B (CALGB 9134) compared neoadjuvant chemotherapy (cisplatin and etoposide) to standard fractionation neoadjuvant radiotherapy (40 Gy in 20 fractions) and was closed prematurely due to slow patient accrual. No difference was found between nodal downstaging, survival or rates of complete surgical resection, and pCR in the radiation arm was $0 \%(40,43)$. Given the increased toxicity of neoadjuvant radiotherapy alone, along with the failure to improve survival outcomes, these studies led to the abandonment of this approach.

\section{Pathologic response after neoadjuvant chemoradiation in locally advanced NSCLC}

The demonstration of a modestly improved OS with the combination of CRT in inoperable patients $(44,45)$ led to a subsequent interest in trimodality therapy with the aim of improving the persistently dismal prognosis of these patients. Several studies of preoperative cisplatinbased chemotherapy and radiotherapy have evaluated the pathologic response in LA-NSCLC and have demonstrated pCR rates varying from $17 \%$ to $45 \%$ (Table 3) (46-53). The most important studies are summarized below.

The phase III randomized control trial of the North American Intergroup trial 0139 (INT0139) compared neoadjuvant CRT (cisplatin, etoposide, plus 45 Gy) followed by surgery 3-5 weeks later $v s$. definitive CRT in patients with T1-3N2M0 NSCLC (51). PCR was achieved in $18 \%$ of 164 patients that underwent a thoracotomy following neoadjuvant CRT (51). The Southwest Oncology Group 9416 trial/North American Intergroup 0160 trial reported 34\% pCR at 3-5 weeks after neoadjuvant CRT consisting in a combination of cisplatin and etoposide delivered concurrently with fractionated radiotherapy to a dose of 45 Gy $(46,47)$. Importantly, the latter trial showed that pCR was associated with improved OS. In fact, at a median follow-up of 84 months, median survival was 30 months in patients with minimal microscopic disease and 29 months in patients with gross residual disease, while it was not reached in patients with pCR $(46,47)$ The subsequent Japanese Clinical Oncology Group 9806 study used concurrent mitomycin, vindesine, cisplatin and radiotherapy to dose of $45 \mathrm{~Gy}$ in superior sulcus tumors, followed by surgical resection at 2-4 weeks and reported 6 -year OS of $56 \%$ and pCR of $21 \%$ (12/57 patients) (48). The Radiation Therapy Oncology group 0229 study attempted curative radiation doses reaching $61 \mathrm{~Gy}$, in combination with carboplatin and paclitaxel, followed by surgery within 8 weeks of CRT completion, but pCR was only $8 \%$, casting doubts on the potential of conventionally fractionated dose escalation to improve $\mathrm{pCR}$ rates (54).

While the above-mentioned studies reported outcomes of standard fractionation radiotherapy, additional studies looked at the role of hyperfractionated radiotherapy combined with chemotherapy to further improve outcomes. Two landmark phase III trials conducted in LA-NSCLC $(55,56)$ suggest that radiation dose escalation through accelerated-hyperfractionation could be a promising 


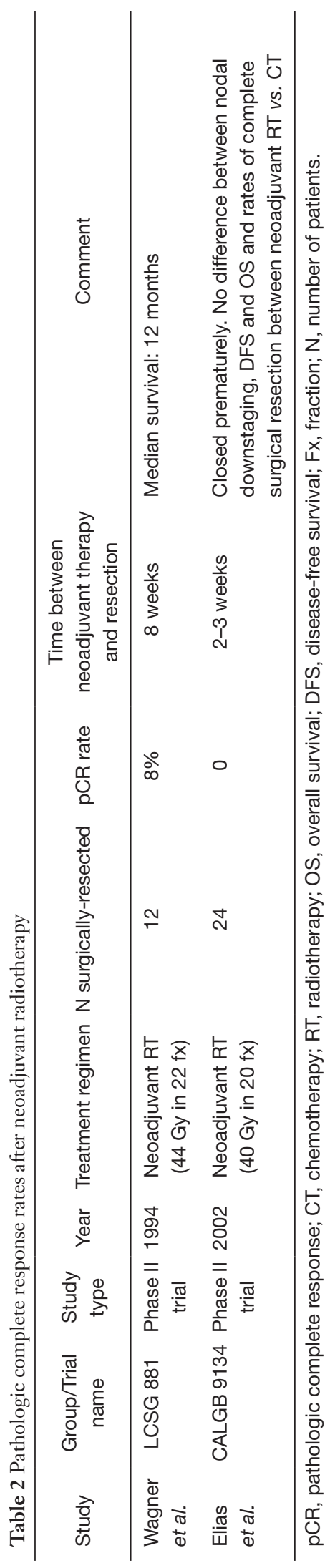

approach to increase pCR rates. The ESPATUE trial evaluated induction chemotherapy (cisplatin and paclitaxel) with concurrent CRT (45 Gy, 1.5 Gy twice daily) in stage IIIA-B NSCLC and showed pCR in 33\% of 81 resected specimens (55). The German Lung Cancer Cooperative Group phase III trial approach consisted of three cycles of cisplatin and etoposide, followed by acceleratedhyperfractionated radiotherapy (45 Gy, 1.5 Gy twice daily) with concurrent carboplatin and vindesine (56). Interestingly, the latter study did not define pCR rates as complete absence of viable tumor cells, but rather defined histopathological response as less than $10 \%$ of residual tumor cells on hematoxylin and eosin stain. At 4-6 weeks post CRT, $60 \%$ of 98 patients had a histopathological response with less than $10 \%$ residual tumor cells (56).

\section{Predicting $p C R$}

PCR analysis may help determine early signs of therapeutic efficacy, years before survival data is available and could determine the necessity for additional adjuvant therapeutic interventions (2). There must be a balance between assessing pCR at an optimal timepoint to ensure it is a representative measure of therapy-induced cell damage, yet without compromising surgical resection and oncologic outcomes (57). From a radiobiological standpoint, we previously discussed that later pCR assessments are more likely to grasp the effect of mitotic death induced by radiotherapy. However, the clinical literature in LA-NSCLC suggests that prolonged time before surgical resection after completion of neoadjuvant therapy may jeopardize cancer outcomes. In fact, a large retrospective study from the National Cancer Database of 1,623 patients with stage IIIA NSCLC found significantly improved survival in patients operated at $0-3$ weeks post-CRT (30\%), compared to those operated at 6-9 weeks $(20 \%, \mathrm{P}=0.04)(58)$. In patients with LA-NSCLC selected for trimodality approach, it is therefore common practice to aim for rapid restaging and resection within 6 weeks from neoadjuvant therapy completion. This being said, it should be restated that the survival advantage of the addition of surgery to concurrent CRT has not been shown, and that the recent results from the PACIFIC trial have established radical CRT and adjuvant immunotherapy as a standard of care in LA-NSCLC. Nonetheless, predicting pCR in LA-NSCLC treated with CRT could be critical for optimal selection of patients who may benefit from additional treatment intensification (52). 
Current literature suggests that symptom improvement (brachial plexus-related) in superior sulcus tumors, preoperative radiation dose over 54 Gy and the percent reduction in tumor size post CRT are associated with $\mathrm{pCR}$ $(52,53)$. Haque et al. retrospectively analysed 1,750 patients from the National Cancer Database from 2004-2015 that underwent neoadjuvant CRT for histologically confirmed T1-4N2M0 NSCLC. This study confirmed the improved prognosis of patients who achieved pCR (median OS in pCR $=72$ months $v s$. others $=40$ months) (53). Though radiation dose over 54 Gy was associated with pCR, doses above 59.4 Gy resulted in higher postoperative mortality, suggesting that increasing radiation dose to augment radiation efficacy may be more toxic and requires careful investigation. Antonoff et al. (52) focused on superior sulcus tumors and found, on univariate analysis, that symptom improvement (abatement of pain or neurological symptoms secondary to compression of the brachial plexus) and tumor size reduction on CT were associated with pCR, but not radiation dose. On multivariate analysis, only tumor size reduction on pre-operative imaging remained an independent predictor of pCR (52). PCR rate among patients with combined reduction in tumor size and improvement in symptoms was as high as $88 \%$.

While robust predictors of pCR are still lacking, advances in the fields of functional imaging, radiomics and liquid-based genomics may aid in the non-invasive assessment of tumor response and prediction of cancer outcomes. In addition to simple anatomic assessments of tumor size, functional imaging can characterize tumor activity by measuring glucose uptake, perfusion, hypoxia and proliferation, all potential indicators of tumor cell viability (59). Furthermore, the emergence of minimally invasive "omic" approaches including radiomics (consisting in the extraction and analysis of quantitative features from radiologic images) (60-62) and liquid-based genomics (circulating tumor DNA and circulating tumor cells) (63), begin to show promise for response assessment in lung cancer; however, their investigation remains embryonic.

\section{The disruptive advent of immunotherapy}

Immune checkpoint blockade has recently completely changed the treatment paradigm in locally advanced and metastatic NSCLC, becoming standard of care as first line therapy in both settings $(64,65)$. In fact, patients with metastatic NSCLC and without EGFR/ALK genomic aberrations are now offered pembrolizumab as first line therapy after demonstration of improved survival compared to platinum-based chemotherapy $(66,67)$. More recently, results from the PACIFIC trial have disrupted the treatment paradigm in LA-NSCLC after showing significantly improved survival at 2 years in patients treated with adjuvant durvalumab after standard CRT (37). Although the increased potency of this new treatment combination suggests that $\mathrm{pCR}$ rates could be higher with the use of immunotherapy, the patterns of response and progression after immunotherapy seem to differ from those observed with conventional therapies, complicating both radiological and pathological assessments (68). A response tool better adapted to immunotherapy was developed in 2017, the immunotherapy Response Evaluation Criteria in Solid Tumors (iRECIST) (69), but radiological assessments after immunotherapy remain challenging, even with the aid of these guidelines (70-72).

Forde et al. conducted the first pilot study of neoadjuvant programmed cell death 1 blockade in resectable lung cancer with nivolumab administered 4 weeks prior to surgery (73). Of 21 patients with stage I-IIIB NSCLC, $45 \%$ had a "major pathologic response" (less than $10 \%$ viable tumor cells on hematoxylin and eosin staining), and $14 \%$ had complete pathologic response as defined by the absence of viable tumor cells (73). It is postulated that radiotherapy could work synergistically with immunotherapy by modifying the immunosuppressive tumor microenvironment and strengthening the immunogenicity of tumor cells by increasing tumor neoantigens, activating dendritic cells and increasing T-cell recruitment (74-77). The advent of immunotherapy has renewed the interest for neoadjuvant therapy followed by surgical resection, as demonstrated by the several on-going trials evaluating the combination of neoadjuvant immune checkpoint inhibitors and radiotherapy +/- chemotherapy in resectable LA-NSCLC patients (NCT03237377, NCT02987998, NCT03871153) (74). The interest for immunotherapy has also been extended to SABR, where current efforts are centered on lowering regional and distant recurrence rates. As such, the combination of radiotherapy and immunotherapy is currently being evaluated in several trials delivering concurrent and adjuvant immunotherapy with SABR (NCT03217071, NCT03446911, NCT03574220, NCT03833154). In short, our current knowledge and understanding of pCR after radiotherapy $+/$ - chemotherapy is limited, but will likely be further complicated and perhaps rendered obsolete with the widespread use of immunotherapy in NSCLC. 


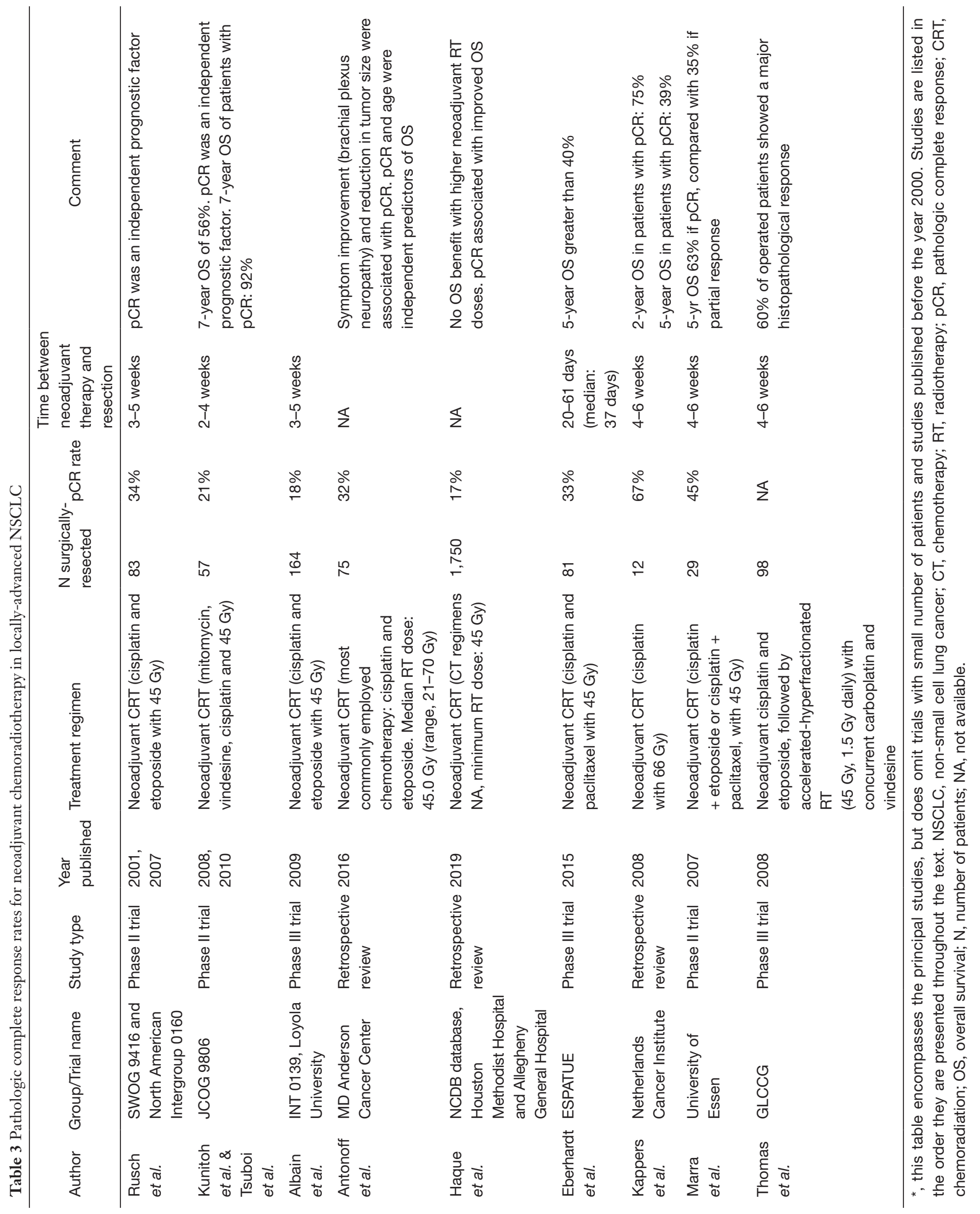




\section{Conclusions}

Evaluating pathologic response rates after lung radiotherapy is highly informative regarding treatment efficacy and patients' prognostication in order to help select patients most in need for treatment intensification. However, assessment of pCR is challenging, given that its definition is not uniform across studies and that the best timing for histological assessment of pCR after both conventional radiotherapy +/chemotherapy and SABR is unclear. Our current response assessment methods, based on radiological evaluations, are certainly not optimal, and further studies correlating radiological and pathological findings as well as exploring the role of predictive biomarkers hold the promise to provide more accurate non-invasive response assessment methods. Finally, our current knowledge of pCR after neoadjuvant treatment will likely be profoundly challenged in the upcoming years by the integration of immunotherapy in the treatment of NSCLC.

\section{Acknowledgments}

None.

\section{Footnote}

Conflicts of Interest: H Bahig received a research grant from Varian Medical Systems and honoraria from Siemens Healthineers and Bristol Myers Squibb. AV Louie has received honoraria from Varian Medical Systems Inc. and AstraZeneca. P Wong has research grants from AstraZeneca and Bristol-Myers Squibb. The other authors have no conflicts of interest to declare.

Ethical Statement: The authors are accountable for all aspects of the work in ensuring that questions related to the accuracy or integrity of any part of the work are appropriately investigated and resolved.

\section{References}

1. Schreiner W, Gavrychenkova S, Dudek W, et al. Pathologic complete response after induction therapy-the role of surgery in stage IIIA/B locally advanced non-small cell lung cancer. J Thorac Dis 2018;10:2795-803.

2. El Kadmiri MAA, Rajan A. Neoadjuvant immunotherapy for non-small cell lung cancer: can early intervention result in durable clinical benefit? J Thorac Dis 2018;10:S3203-6.
3. Hellmann MD, Chaft JE, William WN Jr, et al. Pathological response after neoadjuvant chemotherapy in resectable non-small-cell lung cancers: proposal for the use of major pathological response as a surrogate endpoint. Lancet Oncol 2014;15:e42-50.

4. Pataer A, Kalhor N, Correa AM, et al. Histopathologic response criteria predict survival of patients with resected lung cancer after neoadjuvant chemotherapy. J Thorac Oncol 2012;7:825-32.

5. Betticher DC, Hsu Schmitz SF, Totsch M, et al. Prognostic factors affecting long-term outcomes in patients with resected stage IIIA pN2 non-small-cell lung cancer: 5-year follow-up of a phase II study. Br J Cancer 2006;94:1099-106.

6. Palma DA, Nguyen TK, Louie AV, et al. Measuring the Integration of Stereotactic Ablative Radiotherapy Plus Surgery for Early-Stage Non-Small Cell Lung Cancer: A Phase 2 Clinical Trial. JAMA Oncol 2019;5:681-8.

7. Verstegen NE, Lagerwaard FJ, Hashemi SM, et al. Patterns of Disease Recurrence after SABR for Early Stage Non-Small-Cell Lung Cancer: Optimizing FollowUp Schedules for Salvage Therapy. J Thorac Oncol 2015;10:1195-200.

8. Soldà F, Lodge $M$, Ashley $S$, et al. Stereotactic radiotherapy (SABR) for the treatment of primary nonsmall cell lung cancer; systematic review and comparison with a surgical cohort. Radiother Oncol 2013;109:1-7.

9. Senthi S, Lagerwaard FJ, Haasbeek CJ, et al. Patterns of disease recurrence after stereotactic ablative radiotherapy for early stage non-small-cell lung cancer: a retrospective analysis. Lancet Oncol 2012;13:802-9.

10. Sun B, Brooks ED, Komaki RU, et al. 7-year follow-up after stereotactic ablative radiotherapy for patients with stage I non-small cell lung cancer: Results of a phase 2 clinical trial. Cancer 2017;123:3031-9.

11. Nguyen NP, Garland L, Welsh J, et al. Can stereotactic fractionated radiation therapy become the standard of care for early stage non-small cell lung carcinoma. Cancer Treat Rev 2008;34:719-27.

12. Fakiris AJ, McGarry RC, Yiannoutsos CT, et al. Stereotactic body radiation therapy for early-stage non-small-cell lung carcinoma: four-year results of a prospective phase II study. Int J Radiat Oncol Biol Phys 2009;75:677-82.

13. Timmerman RD, Hu C, Michalski JM, et al. Longterm Results of Stereotactic Body Radiation Therapy in Medically Inoperable Stage I Non-Small Cell Lung Cancer. JAMA Oncol 2018;4:1287-8. 
14. Timmerman RD, Paulus R, Pass HI, et al. Stereotactic Body Radiation Therapy for Operable Early-Stage Lung Cancer: Findings From the NRG Oncology RTOG 0618 Trial. JAMA Oncol 2018;4:1263-6.

15. Frakulli R, Salvi F, Balestrini D, et al. Radiological differential diagnosis between fibrosis and recurrence after stereotactic body radiation therapy (SBRT) in early stage non-small cell lung cancer (NSCLC). Transl Lung Cancer Res 2017;6:S1-7.

16. Huang K, Dahele M, Senan S, et al. Radiographic changes after lung stereotactic ablative radiotherapy (SABR)--can we distinguish recurrence from fibrosis? A systematic review of the literature. Radiother Oncol 2012;102:335-42.

17. Palma DA, van Sornsen de Koste J, Verbakel WF, et al. Lung density changes after stereotactic radiotherapy: a quantitative analysis in 50 patients. Int J Radiat Oncol Biol Phys 2011;81:974-8.

18. Trovo M, Linda A, El Naqa I, et al. Early and late lung radiographic injury following stereotactic body radiation therapy (SBRT). Lung cancer (Amsterdam, Netherlands) 2010;69:77-85.

19. Dahele M, Palma D, Lagerwaard F, et al. Radiological changes after stereotactic radiotherapy for stage I lung cancer. J Thorac Oncol 2011;6:1221-8.

20. Palma DA, Senan S, Haasbeek CJ, et al. Radiological and clinical pneumonitis after stereotactic lung radiotherapy: a matched analysis of three-dimensional conformal and volumetric-modulated arc therapy techniques. Int J Radiat Oncol Biol Phys 2011;80:506-13.

21. Eisenhauer EA, Therasse P, Bogaerts J, et al. New response evaluation criteria in solid tumours: revised RECIST guideline (version 1.1). Eur J Cancer 2009;45:228-47.

22. Huang K, Senthi S, Palma DA, et al. High-risk CT features for detection of local recurrence after stereotactic ablative radiotherapy for lung cancer. Radiother Oncol 2013;109:51-7.

23. Essler M, Wantke J, Mayer B, et al. Positron-emission tomography CT to identify local recurrence in stage I lung cancer patients 1 year after stereotactic body radiation therapy. Strahlenther Onkol 2013;189:495-501.

24. Bollineni VR, Widder J, Pruim J, et al. Residual (1)(8) F-FDG-PET uptake 12 weeks after stereotactic ablative radiotherapy for stage I non-small-cell lung cancer predicts local control. Int J Radiat Oncol Biol Phys 2012;83:e551-5.

25. Nguyen TK, Senan S, Bradley JD, et al. Optimal imaging surveillance after stereotactic ablative radiation therapy for early-stage non-small cell lung cancer: Findings of an
International Delphi Consensus Study. Pract Radiat Oncol 2018;8:e71-8.

26. Scarbrough TJ. Analysis of Pathologic Complete Response 10 Weeks After Radiotherapy-A Radiobiological Sin. JAMA Oncol 2019. [Epub ahead of print].

27. Eriksson D, Stigbrand T. Radiation-induced cell death mechanisms. Tumour Biol 2010;31:363-72.

28. Galluzzi L, Maiuri MC, Vitale I, et al. Cell death modalities: classification and pathophysiological implications. Cell Death Differ 2007;14:1237-43.

29. Seymour CB, Mothersill C, Alper T. High yields of lethal mutations in somatic mammalian cells that survive ionizing radiation. Int J Radiat Biol Relat Stud Phys Chem Med 1986;50:167-79.

30. Bedford JS. Sublethal damage, potentially lethal damage, and chromosomal aberrations in mammalian cells exposed to ionizing radiations. Int J Radiat Oncol Biol Phys 1991;21:1457-69.

31. Glynne-Jones R, Sebag-Montefiore D, Meadows HM, et al. Best time to assess complete clinical response after chemoradiotherapy in squamous cell carcinoma of the anus (ACT II): a post-hoc analysis of randomised controlled phase 3 trial. Lancet Oncol 2017;18:347-56.

32. Pettersson D, Lorinc E, Holm T, et al. Tumour regression in the randomized Stockholm III Trial of radiotherapy regimens for rectal cancer. Br J Surg 2015;102:972-8; discussion 978.

33. Shaikh T, Ruth K, Scott WJ, et al. Increased time from neoadjuvant chemoradiation to surgery is associated with higher pathologic complete response rates in esophageal cancer. Ann Thorac Surg 2015;99:270-6.

34. van der Werf LR, Dikken JL, van der Willik EM, et al. Time interval between neoadjuvant chemoradiotherapy and surgery for oesophageal or junctional cancer: A nationwide study. Eur J Cancer 2018;91:76-85.

35. Song CW, Kim MS, Cho LC, et al. Radiobiological basis of SBRT and SRS. Int J Clin Oncol 2014;19:570-8.

36. Oweida A, Sabri S, Al-Rabea A, et al. Response to stereotactic ablative radiotherapy in a novel orthotopic model of non-small cell lung cancer. Oncotarget 2017;9:1630-40.

37. Antonia SJ, Villegas A, Daniel D, et al. Overall Survival with Durvalumab after Chemoradiotherapy in Stage III NSCLC. N Engl J Med 2018;379:2342-50.

38. Bloedorn FG, Cowley RA, Cuccia CA, et al. Preoperative Irradiation in Bronchogenic Carcinoma. Am J Roentgenol Radium Ther Nucl Med 1964;92:77-87.

39. Bromley LL, Szur L. Combined radiotherapy and 
resection for carcinoma of the bronchus; experiences with 66 patients. Lancet 1955;269:937-41.

40. Elias AD, Kumar P, Herndon J, 3rd, et al. Radiotherapy versus chemotherapy plus radiotherapy in surgically treated IIIA N2 non-small-cell lung cancer. Clin Lung Cancer 2002;4:95-103.

41. Wagner H Jr, Lad T, Piantadosi S, et al. Randomized phase 2 evaluation of preoperative radiation therapy and preoperative chemotherapy with mitomycin, vinblastine, and cisplatin in patients with technically unresectable stage IIIA and IIIB non-small cell cancer of the lung. LCSG 881. Chest 1994;106:348S-54S.

42. Warram J. Preoperative irradiation of cancer of the lung: final report of a therapeutic trial. A collaborative study. Cancer 1975;36:914-25.

43. Yalman D. Neoadjuvant radiotherapy/chemoradiotherapy in locally advanced non-small cell lung cancer. Balkan Med J 2015;32:1-7.

44. Dillman RO, Herndon J, Seagren SL, et al. Improved survival in stage III non-small-cell lung cancer: seven-year follow-up of cancer and leukemia group B (CALGB) 8433 trial. J Natl Cancer Inst 1996;88:1210-5.

45. Curran WJ Jr, Paulus R, Langer CJ, et al. Sequential vs. concurrent chemoradiation for stage III non-small cell lung cancer: randomized phase III trial RTOG 9410. J Natl Cancer Inst 2011;103:1452-60.

46. Rusch VW, Giroux DJ, Kraut MJ, et al. Induction chemoradiation and surgical resection for non-small cell lung carcinomas of the superior sulcus: Initial results of Southwest Oncology Group Trial 9416 (Intergroup Trial 0160). J Thorac Cardiovasc Surg 2001;121:472-83.

47. Rusch VW, Giroux DJ, Kraut MJ, et al. Induction chemoradiation and surgical resection for superior sulcus non-small-cell lung carcinomas: long-term results of Southwest Oncology Group Trial 9416 (Intergroup Trial 0160). J Clin Oncol 2007;25:313-8.

48. Kunitoh H, Kato H, Tsuboi M, et al. Phase II trial of preoperative chemoradiotherapy followed by surgical resection in patients with superior sulcus non-small-cell lung cancers: report of Japan Clinical Oncology Group trial 9806. J Clin Oncol 2008;26:644-9.

49. Tsuboi M, Kunitoh H, Shibata T, et al. Seven-year followup of preoperative chemoradiotherapy in superior sulcus tumor: Report of a Japan Clinical Oncology Group Trial (JCOG9806). J Clin Oncol 2010;28:abstr 7025.

50. Kernstine KH, Moon J, Kraut MJ, et al. Trimodality therapy for superior sulcus non-small cell lung cancer: Southwest Oncology Group-Intergroup Trial S0220. Ann
Thorac Surg 2014;98:402-10.

51. Albain KS, Swann RS, Rusch VW, et al. Radiotherapy plus chemotherapy with or without surgical resection for stage III non-small-cell lung cancer: a phase III randomised controlled trial. Lancet 2009;374:379-86.

52. Antonoff MB, Hofstetter WL, Correa AM, et al. Clinical Prediction of Pathologic Complete Response in Superior Sulcus Non-Small Cell Lung Cancer. Ann Thorac Surg 2016;101:211-7.

53. Haque W, Verma V, Butler EB, et al. Pathologic nodal clearance and complete response following neoadjuvant chemoradiation for clinical N2 non-small cell lung cancer: Predictors and long-term outcomes. Lung Cancer 2019;130:93-100.

54. Suntharalingam M, Paulus R, Edelman MJ, et al. Radiation therapy oncology group protocol 02-29: a phase II trial of neoadjuvant therapy with concurrent chemotherapy and full-dose radiation therapy followed by surgical resection and consolidative therapy for locally advanced non-small cell carcinoma of the lung. Int J Radiat Oncol Biol Phys 2012;84:456-63.

55. Eberhardt WE, Pottgen C, Gauler TC, et al. Phase III Study of Surgery Versus Definitive Concurrent Chemoradiotherapy Boost in Patients With Resectable Stage IIIA(N2) and Selected IIIB Non-Small-Cell Lung Cancer After Induction Chemotherapy and Concurrent Chemoradiotherapy (ESPATUE). J Clin Oncol 2015;33:4194-201.

56. Thomas M, Rube C, Hoffknecht P, et al. Effect of preoperative chemoradiation in addition to preoperative chemotherapy: a randomised trial in stage III non-smallcell lung cancer. Lancet Oncol 2008;9:636-48.

57. Samson P, Crabtree TD, Robinson CG, et al. Defining the Ideal Time Interval Between Planned Induction Therapy and Surgery for Stage IIIA Non-Small Cell Lung Cancer. Ann Thorac Surg 2017;103:1070-5.

58. Gao SJ, Corso CD, Wang EH, et al. Timing of Surgery after Neoadjuvant Chemoradiation in Locally Advanced Non-Small Cell Lung Cancer. J Thorac Oncol 2017;12:314-22.

59. Harry VN, Semple SI, Parkin DE, et al. Use of new imaging techniques to predict tumour response to therapy. Lancet Oncol 2010;11:92-102.

60. Starkov P, Aguilera TA, Golden DI, et al. The use of texture-based radiomics CT analysis to predict outcomes in early-stage non-small cell lung cancer treated with stereotactic ablative radiotherapy. Br J Radiol 2019;92:20180228. 
61. Ahn HK, Lee H, Kim SG, et al. Pre-treatment (18)F-FDG PET-based radiomics predict survival in resected nonsmall cell lung cancer. Clin Radiol 2019;74:467-73.

62. He B, Zhao W, Pi JY, et al. A biomarker basing on radiomics for the prediction of overall survival in nonsmall cell lung cancer patients. Respir Res 2018;19:199.

63. Saarenheimo J, Eigeliene N, Andersen H, et al. The Value of Liquid Biopsies for Guiding Therapy Decisions in Nonsmall Cell Lung Cancer. Front Oncol 2019;9:129.

64. Proto C, Ferrara R, Signorelli D, et al. Choosing wisely first line immunotherapy in non-small cell lung cancer (NSCLC): what to add and what to leave out. Cancer Treat Rev 2019;75:39-51.

65. Pacheco JM, Camidge DR, Doebele RC, et al. A Changing of the Guard: Immune Checkpoint Inhibitors With and Without Chemotherapy as First Line Treatment for Metastatic Non-small Cell Lung Cancer. Front Oncol 2019;9:195.

66. Reck M, Rodriguez-Abreu D, Robinson AG, et al. Pembrolizumab versus Chemotherapy for PD-L1Positive Non-Small-Cell Lung Cancer. N Engl J Med 2016;375:1823-33.

67. Gandhi L, Rodriguez-Abreu D, Gadgeel S, et al. Pembrolizumab plus Chemotherapy in Metastatic NonSmall-Cell Lung Cancer. N Engl J Med 2018;378:2078-92.

68. Borcoman E, Nandikolla A, Long G, et al. Patterns of Response and Progression to Immunotherapy. Am Soc Clin Oncol Educ Book 2018;38:169-78.

69. Al-Bannai R, Miller D, Sadownik L, et al. Vulvar Acanthosis With Altered Differentiation (VAAD): Report of a Case With Progression to Poorly Differentiated Carcinoma Over a 5-Yr Period. Int J Gynecol Pathol

Cite this article as: Roy SF, Louie AV, Liberman M, Wong P, Bahig H. Pathologic response after modern radiotherapy for non-small cell lung cancer. Transl Lung Cancer Res 2019;8(Suppl 2):S124-S134. doi: 10.21037/tlcr.2019.09.05
2015;34:385-9.

70. Vrankar M, Unk M. Immune RECIST criteria and symptomatic pseudoprogression in non-small cell lung cancer patients treated with immunotherapy. Radiol Oncol 2018;52:365-9.

71. Beer L, Hochmair M, Haug AR, et al. Comparison of RECIST, iRECIST, and PERCIST for the Evaluation of Response to PD-1/PD-L1 Blockade Therapy in Patients With Non-Small Cell Lung Cancer. Clin Nucl Med 2019;44:535-43.

72. Song P, Zhang J, Shang C, et al. Curative effect assessment of immunotherapy for non-small cell lung cancer: The "blind area" of Immune Response Evaluation Criteria in Solid Tumors (iRECIST). Thorac Cancer 2019;10:587-92.

73. Forde PM, Chaft JE, Smith KN, et al. Neoadjuvant PD-1 Blockade in Resectable Lung Cancer. N Engl J Med 2018;378:1976-86.

74. Yang H, Jin T, Li M, et al. Synergistic effect of immunotherapy and radiotherapy in non-small cell lung cancer: current clinical trials and prospective challenges. Precision Clinical Medicine 2019;2:57-70.

75. Lin AJ, Roach M, Bradley J, et al. Combining stereotactic body radiation therapy with immunotherapy: current data and future directions. Transl Lung Cancer Res 2019;8:107-15.

76. Ko EC, Raben D, Formenti SC. The Integration of Radiotherapy with Immunotherapy for the Treatment of Non-Small Cell Lung Cancer. Clin Cancer Res 2018;24:5792-806.

77. Bhalla N, Brooker R, Brada M. Combining immunotherapy and radiotherapy in lung cancer. J Thorac Dis 2018; 10:S1447-60. 